\title{
SUBMANIFOLDS OF QUATERNION PROJECTIVE SPACE WITH BOUNDED SECOND FUNDAMENTAL FORM
}

\author{
By Partick Coulton and Hillel Gauchman
}

\begin{abstract}
Let $h$ be the second fundamental form of a compact submanifold $M$ of the quaternion projective space $\boldsymbol{H} \boldsymbol{P}^{n}(1)$. For any unit vector $u \in T M$, set $\delta(u)=\|h(u, u)\|^{2}$. We determine all compact totally complex submanifolds of $\boldsymbol{H P}^{n}$ (1) (resp. all compact totally real minimal submanifolds of $\boldsymbol{H P}^{n}(1)$ ) satisfying condition $\delta(u) \leqq \frac{1}{4}$ (resp. $\left.\delta(u) \leqq \frac{1}{12}\right)$ for all unit vectors $u \in T M$.
\end{abstract}

\section{Introduction.}

Let $M$ be a smooth $m$-dimensional Riemannian manifold isometrically immersed in an $(m+p)$-dimensional Riemannian manifold $\tilde{M}$. Let $h$ denote the second fundamental form of this immersion. For each $x \in M, h$ is a bilinear mapping from $T M_{x} \times T M_{x}$ into $T M_{x}^{\frac{1}{x}}$, where $T M_{x}$ is the tangent space of $M$ at $x$ and $T M_{x}^{\frac{1}{x}}$ is the normal space. We denote by $S(x)$ the square of the length of $h$ at $x \in M$. By Gauss' equation we have $S(x)=m(m-1)-\rho(x)$, whenever $M$ is immersed as a minimal submanifold of $S^{m+p}(1)$ with scalar curvature $\rho(x)$ at $x$ in $M$. Therefore $S(x)$ is an intrinsic invariant of $M$.

In 1968, J. Simons [12] discovered for the class of compact minimal $m$-dimensional submanifolds of the unit $(m+p)$-sphere that the totally geodesic submanifolds are isolated in the following sense: If $S(x)<n /(2-1 / p)$ for all $x \in M$, then $S(x) \equiv 0$ on $M$, and thus $M$ is totally geodesic. In [1], S. S. Chern, $M$. do Carmo, and S. Kobayashi determined all minimal submanifolds of the unit sphere satisfying $S(x) \equiv n /(2-1 / p)$. Later similar results were obtained for various types of minimal submanifolds of the complex projective spaces and the quaternion projective spaces.

Let $T: U M \rightarrow M$ and $U M_{x}$ denote the unit tangent bundle of $M$ along with its fibre over $x \in M$. We set $\delta(u)=\|h(u, u)\|^{2}$ for $u \in U M$. Observe that $\delta(u)$ is not an intrinsic invariant of the submanifold $M$. However, like $S(x), \delta(u)$ can be considered as a natural measure of the degree to which an immersion fails to be totally geodesic.

In [10], and [11], A. Ros proved that if $M$ is a compact Kaehler submanifold of $\boldsymbol{C P} \boldsymbol{P}^{n}(1)$ and if $\delta(u)<1 / 4$, for any $u \in U M$, then $M$ is totally geodesic in

Received May 23, 1988. 
$\boldsymbol{C P}^{n}(1)$. Ros also gives a complete list of Kaehler submanifolds in $\boldsymbol{C P}^{n}(1)$ which satisfy the condition

$$
\max _{u \in U M}\{\delta(u)\}=1 / 4
$$

One of the authors obtained results ([4], [5]) similar to the results of Ros for minimal submanifolds of a sphere and for totally real minimal submanifolds of $\boldsymbol{C P} \boldsymbol{P}^{n}(1)$. In the present paper we obtain analogous results for totally complex and totally real minimal submanifolds of quaternion projective space $\boldsymbol{H} \boldsymbol{P}^{n}(1)$.

Recall the standard totally complex imbeddings [3]:

$$
\tau: \boldsymbol{C P}^{n}(1) \longrightarrow \boldsymbol{H P}^{n}(1),
$$

along with the following standard imbeddings [8]:

$$
\begin{aligned}
& \tilde{\phi}_{1}: \boldsymbol{C} \boldsymbol{P}^{m}(1 / 2) \longrightarrow \boldsymbol{C P}^{k}(1), \quad \text { where } k=m(m+3) / 2 \\
& \tilde{\phi}_{2}: \boldsymbol{C} \boldsymbol{P}^{m-s}(1) \times \boldsymbol{C} \boldsymbol{P}^{s}(1) \longrightarrow \boldsymbol{C P}^{k}(1), \quad k=m+s(m-s) \\
& \tilde{\phi}_{3}: Q^{m} \longrightarrow \boldsymbol{C P}^{m+1}(1), \quad m \geqq 3 \text { and } Q \text { is the standard complex quadric. } \\
& \tilde{\phi}_{4}: U\left(\frac{m+4}{2}\right) / U(2) \times U(m / 2) \longrightarrow \boldsymbol{C P}^{k}(1), \quad k=m(m+10) / 8 \\
& \tilde{\phi}_{5}: S O(10) / U(5) \longrightarrow \boldsymbol{C} \boldsymbol{P}^{15}(1) \\
& \tilde{\phi}_{6}: E_{6} / \operatorname{Spin}(10) \times T \longrightarrow \boldsymbol{C P}^{26}(1) .
\end{aligned}
$$

We define the imbeddings of $\phi_{i}=\tau \circ \tilde{\phi}_{i}$, which we call the Nakagawa-Takagi imbeddings or the $N T$ imbeddings.

THEOREM 1. Let $M$ be a compact totally complex submanifold of real dimension $2 m$, immersed in the quaternion projective space $\boldsymbol{H P}^{n}(1)$. If $\delta(u) \leqq 1 / 4$ for all $u \in U M$, then either

or

(i) $\delta(u) \equiv 0$ and $M$ is totally geodesic in $\boldsymbol{H P}^{n}(1)$,

(ii) $\operatorname{Max}\{\delta(u)\}=1 / 4$ and $M$ is an imbedded submanifold congruent to one of the NT-imbeddings.

Note that the real dimensions of $M$ for the imbeddings $\phi_{1}, \phi_{2}, \cdots, \phi_{6}$ are $2 m, 2 m, 2 m, 2 m, 20$ and 32 respectively.

THEOREM 2. Let $\phi: M \rightarrow \boldsymbol{H P}^{n}(1)$ be a totally complex immersion of a compact Kaehler manifold $M$ into $\boldsymbol{H P}^{n}(1)$. Let $H$ denote the holomorphic sectional curvature of $M$. If $H>1 / 2$, then $M$ is totally geodesic. If $H \geqq 1 / 2$ and $M$ is not totally geodesic, then $\phi$ is congruent to one of the six NT-imbeddings.

Recall the totally real imbeddings [2]: 


$$
\nu: \boldsymbol{R P}^{n}(1 / 4) \longrightarrow \boldsymbol{H P}^{n}(1),
$$

and the first standard imbeddings of projective spaces:

$$
\begin{aligned}
& \tilde{\psi}_{1}: \boldsymbol{R} \boldsymbol{P}^{2}(1 / 12) \longrightarrow \boldsymbol{R} \boldsymbol{P}^{4}(1 / 4) \\
& \tilde{\psi}_{2}: \boldsymbol{C P}^{2}(1 / 3) \longrightarrow \boldsymbol{R P}^{7}(1 / 4) \\
& \tilde{\psi}_{3}: \boldsymbol{H} \boldsymbol{P}^{2}(1 / 3) \longrightarrow \boldsymbol{R} \boldsymbol{P}^{13}(1 / 4) \\
& \tilde{\psi}_{4}: \operatorname{Cay}^{2}(1 / 3) \longrightarrow \boldsymbol{R} \boldsymbol{P}^{25}(1 / 4) .
\end{aligned}
$$

THEOREM 3. Let $M$ be a compact totally real minimal submanifold of dimension $m$, immersed in the quaternion projective space $\boldsymbol{H P}^{n}(1)$. If $\delta(u) \leqq 1 / 12$ for all $u \in U M$, then either

(i) $\delta(u) \equiv 0$ and $M$ is totally geodesic in $\boldsymbol{H P}^{n}(1)$

or

(ii) $\operatorname{Max}\{\delta(u)\}=1 / 12$ and $M$ is either congruent to one of the imbeddings $\phi_{i}=\nu \circ \tilde{\psi}_{\imath}$ or to the immersion $\phi_{5}=\phi_{1} \circ \pi$, where $\pi: S^{2}(1 / 12) \rightarrow \boldsymbol{R P}^{w}(1 / 12)$ $i$ s the covering map.

Note that the dimension of $M$ for the mappings $\phi_{1}, \phi_{2}, \phi_{3}, \phi_{4}, \psi_{5}$ are $2,4,8,16$, and 2 respectively.

\section{Quaternion Kaehler Manifolds.}

Let $N$ be a differentiable manifold of dimension $4 n$, and assume that there is a 3-dimensional vector bundle $V,[6]$, consisting of tensors of type $(1,1)$ over $N$ satisfying the following condition: in any coordinate neighborhood $U$ of $N$ there is a local base $\{I, J, K\}$ of $V$ called a canonical local base of $V$, such that

$$
\begin{aligned}
& I^{2}=J^{2}=K^{2}=-I d \\
& I J=-J I=K ; \quad J K=-K J=I ; \quad K I=-K I=J,
\end{aligned}
$$

where $I d$ denotes the identity tensor field of type $(1,1)$. If $N$ is a manifold and $V$ is a bundle over $N$ satisfying the above condition then $(N, V)$ is called an almost quaternion manifold. If $g$ is a Riemannian metric for $(N, V)$ such that $g(\phi X, Y)+g(X, \phi Y)=0$, holds for any cross section $\phi$ of $V$, with $X, Y \in T N$, then $(N, V, g)$ is called an almost quaternion metric manifold.

Assume that the Riemannian connection $\nabla$ of $(N, V, g)$ satisfies the following condition: if $\phi$ is a local cross section of the bundle $V$, then $\nabla_{X} \phi$ is also a local cross section of $V$, where $X$ is an arbitrary vector field. In this case $N=(N, V, g)$ is called a Kaehler quaternion manifold.

Let $x \in N$ and $X \in T N_{x}$. Consider the 4-dimensional subspace $Q(x)$ in $T N_{x}$ defined by 


$$
Q(X)=\operatorname{Span}_{R}\{X, I X, J X, K X\} \text {. }
$$

We call this the $Q$-section generated by $X$. If for all $x \in N$, and $X \in T N_{x}$, and $Y, Z \in Q(X)$, the sectional curvature $\sigma(Y, Z)=c$ (a constant), then we say that $N$ is a Kaehler quaternion manifold of constant $Q$-sectional curvature $c$. In addition, such a manifold is called a quaternion space-form.

The curvature operator $R$ of a quaternionic space-form $N=(N, V, g)$ has the form:

$$
R(X, Y) Z=\frac{c}{4}[\Lambda(Y, Z) X-\Lambda(X, Z) Y-2 \Gamma(X, Y) Z]
$$

where $c$ is the $Q$-sectional curvature,

and

$$
\Lambda(Y, Z) X=g(Y, Z) X+g(I Y, Z) I X+g(J Y, Z) J X+g(K Y, Z) K X
$$

$$
\Gamma(X, Y) Z=g(I X, Y) I Z+g(J X, Y) J Z+g(K X, Y) K Z .
$$

It is well known that the quaterion projective space $\boldsymbol{H} \boldsymbol{P}^{n}(c)$ is a compact $4 n$-dimensional quaternion space-form.

\section{Totally Complex Submanifolds.}

Let $(\tilde{M}, V, \tilde{g})$ be a Kaehler quaternion manifold and let $M$ be a Riemannian manifold immersed in $\tilde{M}$ isometrically by $F: M \rightarrow \tilde{M}$. A submanifold $M$ is called a totally complex submanifold of $\tilde{M}$ [3], if the following two conditions are satisfied:

(i) There exists a global section I of $F^{*}(V)$ satisfying

$$
\tilde{\nabla}_{X} I=0
$$

for any $X \in T M$.

(ii) For each $x \in M$, there exists a neighborhood $U(x) \subset M$ and a canonical local base $\{I, J, K\}$ of $F^{*}(V)$ over $U(x)$ adapted to $I$ such that

$$
I\left(T M_{y}\right)=T M_{y} ; \quad J\left(T M_{y}\right) \perp T M_{y} ; \quad K\left(T M_{y}\right) \perp T M_{y}
$$

for each $y \in U(x)$.

It follows from this definition, that any totally complex submanifold of a Kaehler quaternion manifold is even dimensional. In fact, it is easy to see that it has a natural Kaehler structure. Let $h$ be the second fundamental form of $M$. We define

and

$$
T_{1}(X, Y, Z)=\tilde{g}(h(X, Y), J Z),
$$

$$
T_{2}(X, Y, Z)=\tilde{g}(h(X, Y), K Z)
$$

for $X, Y, Z \in T M_{x}, x \in M$. To simplify notation, we henceforth write $\tilde{g}()=,\langle$,$\rangle .$ 
LEMMA 3.1, [13]. Assume that $M$ is a totally complex submanifold of a Kaehler quaternion manifold then

(i ) $h(I X, Y)=h(X, I Y)=\operatorname{Ih}(X, Y)$

for $X, Y \in T M_{x}, x \in M$.

(ii) $T_{1}$ and $T_{2}$ are symmetric with respect to all three arguments.

(iii) $T_{i}(I X, Y, Z)=T_{i}(X, I Y, Z)=T_{i}(X, Y, I Z)$ for $i=1,2$, and for $X, Y, Z \in T M_{x}, x \in M$.

By Lemma 3.1, $h(I X, I Y)=-h(X, Y)$. It follows that any totally complex submanifold of Kaehler quaternion manifold is minimal. We shall need the following to prove Theorem 1.

LEMMA 3.2, [11]. Let $S$ be a k-covariant tensor field on a compact Riemannian manifold $N$. Then

$$
\int_{U N}(\nabla S)(u, \cdots, u ; u) d u=0,
$$

where $\nabla$ is the Riemann connection on $N, U N$ is the unit tangent bundle of $N$, and $d u$ is the canonical volume element on $U N$.

For the remainder of this section we shall assume that $M$ is a totally complex compact submanifold of real dimension $2 m$ in the quaternionic projective space $\boldsymbol{H} \boldsymbol{P}^{n}(1)$. We shall denote by $\tilde{\nabla}, \nabla$ and $\nabla^{\perp}$ the Riemannian connections on $\boldsymbol{H} \boldsymbol{P}^{n}$, on $M$, and the normal connection on $M$, respectively. We recall that $\delta(u)=\|h(u, u)\|^{2}$, where $u \in U M$.

Lemma 3.3. Assume that $\delta(u) \leqq 1 / 4$ for all $u \in U M$. Then

(i) $\tilde{\nabla} h \equiv 0, \quad$ (i.e. the second fundamental form is parallel).

(ii) $\tilde{g}(h(X, Y), J Z)=\tilde{g}(h(X, Y), K Z)=0$ for all $X, Y, Z \in T M_{x}, x \in M$.

Proof. We shall use the method of Ros [11]. The first and second covariant derivatives of $h$ are given by

and

$$
(\tilde{\nabla} h)(X, Y ; Z)=\nabla_{\frac{1}{Z}}^{\frac{1}{2}}(h(X, Y))-h\left(\nabla_{Z} X, Y\right)-h\left(X, \nabla_{Z} Y\right),
$$

$$
\begin{aligned}
\left(\tilde{\nabla}^{2} h\right)(X, Y ; Z ; W)= & \nabla_{W}^{\perp}((\tilde{\nabla} h)(X, Y ; Z))-(\tilde{\nabla} h)\left(\nabla_{W} X, Y ; Z\right) \\
& -(\tilde{\nabla} h)\left(X, \nabla_{W} Y ; Z\right)-(\tilde{\nabla} h)\left(X, Y ; \nabla_{W} Z\right) .
\end{aligned}
$$

Using equation (2.2), we can write the Codazzi equation as:

$$
(\tilde{\nabla} h)\left(X_{1}, X_{2}, X_{3}\right)=(\tilde{\nabla} h)\left(X_{\sigma(1)}, X_{\sigma(2)}, X_{\sigma(3)}\right)
$$

for any permutation $\sigma$, and for any $X_{1}, X_{2}, X_{3} \in T M_{x}, x \in M$, (i.e. $(\tilde{\nabla} h)$ is symmetric in all three arguments). We obtain the following Ricci identity: 


$$
\left(\tilde{\nabla}^{2} h\right)(X, Y ; Z ; W)-\left(\tilde{\nabla}^{2} h\right)(X, Y ; W ; Z)
$$

$$
=-R^{\perp}(Z, W) h(X, Y)+h(R(Z, W) X, Y)+h(X, R(Z, W) Y),
$$

where $R$ and $R^{\perp}$ denote the curvature tensors associated with $\nabla$ and $\nabla^{\perp}$, respectively. Since $M$ has a Kaehler structure, we have

$$
I R(X, I X) X=R(X, I X) I X \text {. }
$$

Let $t$ be the 4-covariant tensor field on $M$ defined by

$$
t(X, Y, Z, W)=\langle h(X, Y), h(Z, W)\rangle .
$$

Now, for any $u \in U M$, we have

and

$$
(\nabla t)(u, u, u, u ; u)=2\langle(\tilde{\nabla} h)(u, u ; u), h(u, u)\rangle
$$

$$
\begin{aligned}
& \left(\nabla^{2} t\right)=(u, u, u, u ; u ; u) \\
& =2\left\langle\left(\tilde{\nabla}^{2} h\right)(u, u ; u ; u), h(u, u)\right)+2\|(\tilde{\nabla} h)(u, u ; u)\| .
\end{aligned}
$$

Using equations (3.1) through (3.5) and applying Lemma 3.1, we obtain:

$$
\begin{aligned}
& \left(\nabla^{2} t\right)(I u, I u, I u, I u ; I u ; I u) \\
& =2\left\langle\left(\tilde{\nabla}^{2} h\right)(I u, u ; u ; I u), h(u, u)\right\rangle+2\|(\tilde{\nabla} h)(u, u ; u)\|^{2} \\
& =2\left\langle\left(\tilde{\nabla}^{2} h\right)(I u, u ; I u, u), h(u, u)\right\rangle+2\left\langle R^{\perp}(I u, u) I h(u, u), h(u, u)\right\rangle \\
& \quad-4\left\langle R(I u, u) I u, A_{h(u, u)} u\right\rangle+2\|(\tilde{\nabla} h)(u, u ; u)\|^{2} .
\end{aligned}
$$

By Lemma 3.1,

$$
A_{1 \xi}=I A_{\xi}=-A_{\xi} I \text {. }
$$

Using the Ricci equation, (2.2), and (3.7), we obtain

$$
\begin{aligned}
& \left\langle R^{\perp}(I u, u), I h(u, u), h(u, u)\right\rangle \\
& =-\frac{1}{2}\|h(u, u)\|^{2}-2\left\|A_{h(u, u)}(u)\right\|^{2}+\frac{1}{2}\langle h(u, u), J u\rangle^{2}+\frac{1}{2}\langle h(u, u), K u\rangle^{2} .
\end{aligned}
$$

Now, by Gauss' equation and using (2.2) and (3.7) we have

$$
\left\langle R(I u, u) I u, A_{h(u, w)}(u)\right\rangle=-\|h(u, u)\|^{2}+2\left\|A_{h(u, u)}(u)\right\|^{2} .
$$

It follows from (3.2), (3.6), (3.8) and (3.9) that

$$
\begin{aligned}
& \left(\nabla^{2} t\right)(I u, I u, I u, I u ; I u ; I u) \\
& =-2\left\langle\left(\tilde{\nabla}^{2} h\right)(u, u ; u ; u), h(u, u)\right\rangle+3\|h(u, u)\|^{2}-12\left\|A_{h(u, v)}(u)\right\|^{2} \\
& \quad+\langle h(u, u), J u\rangle^{2}+\langle h(u, u), K u\rangle^{2}+2\|(\tilde{\nabla} h)(u, u ; u)\|^{2} .
\end{aligned}
$$


Taking the sum of (3.5) and (3.10), we obtain

$$
\begin{aligned}
& \left(\nabla^{2} t\right)(u, u, u, u ; u, u)+\left(\nabla^{2} t\right)(I u, I u, I u, I u ; I u ; I u) \\
& =3\left(\|h(u, u)\|^{2}-4\left\|A_{h(u, u)}(u)\right\|^{2}\right)+\langle h(u, u), J u\rangle^{2} \\
& \quad+\langle h(u, u), K u\rangle^{2}+4\|\tilde{\nabla} h(u, u ; u)\|^{2} .
\end{aligned}
$$

Integrating (3.11) over $U M$ and applying Lemma 3.2, we have

$$
\begin{aligned}
& 3 \int_{U M}\left(\|h(u, u)-4\| A_{h(u, u)}(u) \|^{2}\right) d u \\
& \quad+\int_{U M}\left(\langle h(u, u), J u\rangle^{2}+\langle h(u, u), K u\rangle^{2} d u+4 \int_{U M}\|\tilde{\nabla} h(u, u ; u)\|^{2} d u=0 .\right.
\end{aligned}
$$

Now observe that by the hypothesis of this lemma $\|h(u, u)\| \leqq 1 / 4$, hence by Schwartz' inequality:

$$
\left\|A_{\xi}(u)\right\|^{2} \leqq\left(\text { maximal eigenvalue of } A_{\xi}\right)^{2} \leqq 1 / 4 \quad(\|\xi\|=1) .
$$

Therefore,

$$
\|h(u, u)\|^{2}-4\left\|A_{h(u, u)}(u)\right\|^{2}=\|h(u, u)\|\left(1-4\left\|A_{\xi} u\right\|^{2}\right) \geqq 0
$$

where $h(u, u)=\|h(u, u)\| \xi$. It now follows from (3.12) that

and

$$
\langle h(u, u), J u\rangle=\langle h(u, u), K u\rangle=0
$$

$$
(\tilde{\nabla} h)(u, u ; u)=0
$$

for each $u \in U M$. Now, using Lemma 3.1 and equation (3.2), we obtain by polarization

and

$$
\langle h(X, Y), J Z\rangle=\langle h(X, Y), K Z\rangle=0,
$$

$$
(\tilde{\nabla} h)(X, Y ; Z)=0 \text {, }
$$

for each $X, Y, Z \in T M_{x}, x \in M$. This completes the proof of the lemma.

Proof of Theorem 1. By Lemma 3.3(i) $M$ has a parallel second fundamental form. All submanifolds of $\boldsymbol{H P}^{n}(1)$ which have parallel second fundamental form have been classified by K. Tsukada in [13]. Lemma 3.3(ii) shows that if the submanifold $M$ in Theorem 1 is not totally geodesic, then it is of the type $(\mathrm{C}-\mathrm{C})$ in Tsukada's classification ([13], Proposition 3.2). It follows from the classification in [13], that the complete list of all submanifolds of the type $(\mathrm{C}-\mathrm{C})$ with parallel second fundamental form is given by the $N T$ imbeddings $\phi_{i}, i=1, \cdots, 6$. It is known that for each $N T$ imbedding

$$
\max _{u \in U M}\{\delta(u)\}=1 / 4 \text {. }
$$


Moreover, this maximum is achieved at every point of $M$. This completes the proof of Theorem 1 .

Proof of Theorem 2. By (2.2) and Gauss' equation we have

$$
H(u)=\langle R(u, I u) I u, u\rangle=1-2 \delta(u),
$$

for any $u \in U M$. Hence the conditions $H(u) \geqq 1 / 2$ is equivalent to the condition $\delta(u) \leqq 1 / 4$. This proves the theorem.

\section{Maximal directions.}

Let $M$ be a compact $m$-dimensional Riemannian manifold isometrically immersed in an $(m+p)$-dimensional Riemannian manifold. As in the previous section we let $h$ denote the second fundamental form, and we define $\delta(u)$ by $\delta(u)=\|h(u, u)\|^{2}$ for $u \in U M$. Assume that for some $u \in U M_{x}$, we have

$$
\delta(u)=\max _{v \in U M}\{\delta(u)\},
$$

then we say that $u$ is a maximal direction at $x \in M$. We say that an orthonormal frame $\left\{e_{1}, \cdots, e_{m+p}\right\}$ is adapted, if $\left\{e_{1}, \cdots, e_{m}\right\}$ is a frame for $T M$, and $\left\{e_{m+1}, \cdots, e_{m+p}\right\}$ is a frame for $T M^{\perp}$. Whenever $\left\{e_{1}, \cdots, e_{m+p}\right\}$ is an adapted frame we use the notation:

$$
h_{\imath j}=h\left(e_{\imath}, e_{j}\right) \quad i, j=1, \cdots, m .
$$

LEMMA 4.1, [5]. If $\left\{e_{1}, \cdots, e_{m+p}\right\}$ is an adapted frame at $x \in M$ such that $e_{1}$ is a maximal direction at $x$, then

$$
\left\langle h_{11}, h_{1 \imath}\right\rangle=0 \quad i=2,3, \cdots, m
$$

where $\langle$,$\rangle denotes \tilde{g}($, ) in $\tilde{M}$.

COROLlaRY. Diagonalizing the symmetric bilinear form $b(X, Y)=\left\langle h_{11}, h(X, Y)\right\rangle$, we can always find an adapted frame $\left\{e_{1}, \cdots, e_{m+p}\right\}$ such that

$$
\begin{aligned}
& e_{1} \text { is a given maximal direction at } x, \\
& \left\langle h_{11}, h_{\imath \jmath}\right\rangle=0, \quad i \neq j, i, j=1,2, \cdots, m .
\end{aligned}
$$

LEMMA 4.2 [5] (Variational Inequality). For any adapted frame satisfying conditions (4.2) and (4.3),

$$
\left\|h_{11}\right\|^{2}-\left\langle h_{11}, h_{i i}\right\rangle-2\left\|h_{1 i}\right\|^{2} \geqq 0, \quad i=2,3, \cdots, m .
$$

Let us define a 4-covariant tensor field $t$ on $M$ by the formula

$$
t(X, Y, Z, W)=\langle h(X, Y), h(Z, W\rangle),
$$


where $X, Y, Z, W \in T M_{x}, x \in M$. The following result is a cosequence of $J$. Simon's formula for $\Delta h,([12],[1])$.

LEMMA 4.3 [5]. For any adapted frame satisfying conditions (4.2) and (4.3) we have

$$
\begin{aligned}
& \frac{1}{2}(\Delta t)\left(e_{1}, e_{1}, e_{1}, e_{1}\right) \\
& =\sum_{i=1}^{m}\left[4\left\langle\tilde{R}\left(e_{1}, e_{2}\right) h_{11}, h_{12}\right\rangle+\left\langle\tilde{R}\left(e_{2}, h_{11}\right) e_{2}, h_{11}\right\rangle-\left\langle h_{11}, h_{i i}\right\rangle^{2}\right. \\
& \quad+2\left(\left\|h_{11}\right\|^{2}-\left\langle h_{11}, h_{i i}\right\rangle\right)\left(\left\langle\tilde{R}\left(e_{1}, e_{2}\right) e_{2}, e_{1}\right\rangle-\left\|h_{1 i}\right\|^{2}\right) \\
& \left.\quad+\left\|(\tilde{\nabla} h)\left(e_{1}, e_{1} ; e_{2}\right)\right\|^{2}\right]+m\left\langle\tilde{R}\left(e_{1}, h_{11}\right) e_{1}, H\right\rangle+m\left\|h_{11}\right\|^{2}\left\langle h_{11}, H\right\rangle,
\end{aligned}
$$

where $\Delta$ is the Laplace operator, $\tilde{R}$ is the curvature tensor of $\tilde{M}, H$ is the mean curvature vector.

Let $s$ be a $k$-covariant tensor field on $M$. Suppose that $u \in U M_{x}$ satisfies

$$
s(u, \cdots, u)=\max _{v \in U M_{x}}\{s(v, \cdots, v)\} .
$$

In such a case we say that $u$ is a maximal direction for $s$ at $x$. For any $x \in M$, we define

$$
f_{s}(x)=s(u, \cdots, u)
$$

where $u$ is a maximal direction for $s$ at $x$. The following result is an obvious generalization of [7], (Proposition 3.1).

LEMMA 4.4 [5] (Generalized Bochner's Lemma). Let $M$ be a compact Riemannian manifold and $s$ a $k$-covariant tensor field on $M$. If

$$
(\Delta s)(u, \cdots, u) \geqq 0
$$

for any maximal direction for $s$, then $f_{s}$ is constant on $M$, and $(\Delta s)(u, \cdots, u)=0$ for any maximal direction $u$ for the tensor s.

\section{Totally Real Minimal Submanifolds.}

Let $\tilde{M}=(\tilde{M}, V, \tilde{g})$ denote a quaternion Kaehler manifold and $M$ be a Riemannian submanifold isometrically immersed in $\tilde{M}$. We say that $M$ is a totally real submanifold of $\tilde{M}$, [2], if

$$
\theta\left(T M_{x}\right) \perp T M_{x}
$$

for any $x \in M$, and any $\theta \in V_{x}$, where $V_{x}$ is the fibre of $V$ over $x$. Recall that $h$ is the second fundamental form, and set

$$
T_{1}(X, Y, Z)=\langle h(X, Y), I Z\rangle
$$




$$
\begin{aligned}
& T_{2}(X, Y, Z)=\langle h(X, Y), J Z\rangle \\
& T_{3}(X, Y, Z)=\langle h(X, Y), K Z\rangle
\end{aligned}
$$

where $\langle$,$\rangle denotes the metric \tilde{g}($,$) .$

LEMMA 5.1 [13]. $T_{i}(X, Y, Z)$ is symmetric in all three arguments for each $i=1,2,3$.

Proof of Theorem 3. Let $x \in M$ and let $\{I, J, K\}$ denote a cannonical local base of $V$ defined in some neighborhood $U(x) \subset \boldsymbol{H P}^{n}(1)$. Let $u$ denote a maximal direction for $t$ at $x$, and let $\left\{e_{1}, \cdots, e_{4 n}\right\}$ denote an adapted frame at $x$ satisfying conditions (4.2) and (4.3). In addition assume that if $w$ is an element of the frame $\left\{e_{1}, \cdots, e_{4 n}\right\}$, then $I w, J w, K w$ are also elements of this frame. Using equation (2.2), Lemma 5.1 and the minimality condition $H=0$, we can rewrite (4.6) in the following form:

$$
\begin{aligned}
& \frac{1}{2}(\Delta t)\left(e_{1}, e_{1}, e_{1}, e_{1}\right) \\
= & 3 m\left\|h_{11}\right\|^{2}\left(\frac{1}{12}-\left\|h_{11}\right\|^{2}\right)+\sum_{\imath=1}^{m}\left(\left\|h_{11}\right\|^{2}-\left\langle h_{11}, h_{i i}\right\rangle\right)\left(\left\|h_{11}\right\|^{2}-\left\langle h_{11}, h_{i i}\right\rangle-2\left\|h_{1 i}\right\|^{2}\right) \\
& +2 \sum_{\imath=1}^{m}\left(\left\|h_{11}\right\|^{4}-\left\langle h_{11}, h_{\imath \imath}\right\rangle^{2}\right)+\frac{1}{4} \sum_{i=1}^{m}\left(\left\langle h_{11}, I e_{\imath}\right\rangle^{2}+\left\langle h_{11}, J e_{\imath}\right\rangle^{2}+\left\langle h_{11}, K e_{\imath}\right\rangle^{2}\right) \\
& +\sum_{\imath=1}^{m}\left\|(\tilde{\nabla} h)\left(e_{1}, e_{1} ; e_{\imath}\right)\right\|^{2} .
\end{aligned}
$$

Now, since $\delta(u) \leqq 1 / 12$ for any $u \in U M$, we have that $\left\|h_{11}\right\|^{2} \leqq 1 / 12$. Therefore, using the Cauchy-Schwartz inequality along with the variational inequality (4.4) we have that each term on the right hand side in (5.1) is non-negative. By Lemma 4.4, $(\Delta t)\left(e_{1}, e_{1}, e_{1}, e_{1}\right)=0$. Hence

$$
\begin{aligned}
& \left\|h_{11}\right\|^{2}\left(\frac{1}{12}-\left\|h_{11}\right\|^{2}\right)=0 ; \\
& \left.\left\|h_{11}\right\|^{2}-\left\langle h_{11}, h_{i i}\right\rangle\right)\left(\left\|h_{11}\right\|^{2}-\left\langle h_{11}, h_{i i}\right\rangle-2\left\|h_{1 i}\right\|^{2}\right)=0, \quad i=2, \cdots, m ; \\
& \left\|h_{11}\right\|^{4}-\left\langle h_{11}, h_{i i}\right\rangle^{2}=0, \quad i=2, \cdots, m ; \\
& \left\langle h_{11}, I e_{\imath}\right\rangle=\left\langle h_{11}, J e_{\imath}\right\rangle=\left\langle h_{11}, K e_{\imath}\right\rangle=0, \quad i=1, \cdots, m ; \\
& (\tilde{\nabla} h)\left(e_{1}, e_{1} ; e_{\imath}\right)=0, \quad i=1, \cdots, m .
\end{aligned}
$$

Now, if $\delta(u)<1 / 12$ for all $u \in U M$, then $h_{11}=0$ by (5.2), and we conclude that $M$ is totally geodesic. Assume, therefore, that

$$
\max _{u \in U M} \delta(u)=1 / 12,
$$

then $\left\|h_{11}\right\|=1 / \sqrt{12}$. By (5.4), we have 


$$
\left\|h_{11}\right\|^{4}=\left\langle h_{11}, h_{i i}\right\rangle^{2} \leqq\left\|h_{11}\right\|^{2}\left\|h_{i i}\right\|^{2} \leqq\left\|h_{11}\right\|^{4} .
$$

Hence, $h_{i i}= \pm h_{11}$ for each $i=1, \cdots, m$. By assumption $M$ is minimal and therefore $m$ is even, $m=2 r$. After a suitable renaming of indices we can write

$$
h_{11}=h_{22}=\cdots=h_{r r}=-h_{r+1, r+1}=\cdots=-h_{2 r, 2 r} \text {. }
$$

Assume that $1 \leqq \lambda, \mu, \nu, \xi \leqq r$, and let $\bar{\lambda}=\lambda+r$, then

$$
h_{\lambda \lambda}=h_{11}, h_{\bar{\lambda} \bar{\lambda}}=-h_{11} \text {. }
$$

Applying equations (4.4) and (5.7) we obtain that $h_{1 \lambda}=0, \lambda \neq 1$. In addition equation (5.7) implies that each element of the frame, $e_{\imath}$, is a maximal direction for $\delta$. Consequently,

$$
h_{\lambda \mu}=h_{\bar{\lambda} \bar{\mu}}=0, \quad \lambda \neq \mu .
$$

Using equations (5.7) and (5.3) we have $\left\|h_{1} \bar{\lambda}\right\|^{2}=\left\|h_{11}\right\|^{2}$, therefore

$$
\left\|h_{\lambda \bar{\mu}}\right\|^{2}=\left\|h_{11}\right\|^{2}=1 / 12 \text {. }
$$

Now since $e_{\imath}$ is a maximal direction for each $i$, we have

$$
\left\|h\left(e_{1}+\tau \sum_{i=2}^{m} x^{\imath} e_{\imath}, e_{1}+\tau \sum_{i=2}^{m} x^{\imath} e_{\imath}\right)\right\|^{2} \leqq\left(1+\sum_{i=2}^{m}\left(x^{i}\right)^{2} \tau^{2}\right)^{2}\left\|h_{11}\right\|^{2}
$$

for $\tau, x^{2}, \cdots, x^{m} \in \boldsymbol{R}$. Expanding in terms of $\tau$ and using equations (4.3), (5.8), and (5.9), we obtain that

$$
-4 \tau^{2} \sum_{\bar{\lambda} \neq \bar{\mu}}\left\langle h_{1 \bar{\lambda}}, h_{1 \bar{\mu}}\right\rangle x^{\bar{\lambda}} x^{\bar{\mu}}+0\left(\tau^{3}\right) \leqq 0
$$

for all real $\tau, x^{2}, \cdots, x^{m}$. Hence $\left\langle h_{1 \bar{\lambda}}, h_{1 \bar{\mu}}\right\rangle=0, \bar{\lambda} \neq \bar{\mu}$. Since each direction $e_{\imath}$ is maximal, we have

$$
\left\langle h_{\lambda \bar{\mu}}, h_{\lambda \bar{\nu}}\right\rangle=0, \quad \bar{\mu} \neq \bar{\nu} ; \quad\left\langle h_{\lambda \bar{\nu}}, h_{\mu \bar{\nu}}\right\rangle=0, \quad \lambda \pm \mu .
$$

Once more expanding (5.10) in terms of $\tau$ we find that

$$
\tau^{3} \sum_{\imath, k \neq 1}\left\langle h_{1 \imath}, h_{j k}\right\rangle x^{\imath} x^{\jmath} x^{k}+0\left(\tau^{4}\right) \leqq 0 .
$$

Hence, $\left\langle h_{1 \imath}, h_{j k}\right\rangle+\left\langle h_{1 \jmath}, h_{k \imath}\right\rangle+\left\langle h_{1 k}, h_{\imath \jmath}\right\rangle=0, i, j, k \neq 1$. By (5.7), (5.8), (5.11), and since each $e_{2}$ is a maximal direction, we obtain

$$
\left\langle h_{\lambda \bar{\nu}}, h_{\mu \bar{\xi}}\right\rangle+\left\langle h_{\lambda \bar{\xi}}, h_{\mu \bar{\nu}}\right\rangle=0 \text {, }
$$

where either $\lambda \neq \mu$ or $\bar{\nu} \neq \bar{\xi}$. Using (4.3), (5.7)-(5.9), (5.11), and (5.12), we obtain by direct computation that $\delta(u)=1 / 12$ for any $u \in U M$. B. O'Neill [9], calls an immersion $\lambda$-isotropic if $\|h(u, u)\|=\lambda$ for any $u \in U M$. Therefore, the immersion under consideration is $\sqrt{1 / 12}$-isotropic.

By (5.6), $(\tilde{\nabla} h)(X, X ; Y)=0$. Using polarization we obtain 


$$
(\tilde{\nabla} h)(X, Y, Z)=0,
$$

for $X, Y, Z \in T M_{x}, x \in M$. Using equation (5.5), and applying polarization, we obtain

$$
\langle h(X, Y), I Z\rangle=\langle h(X, Y), J Z\rangle=\langle h(X, Y), K Z\rangle=0,
$$

for $X, Y, Z \in T M_{x}, x \in M$.

The second fundamental form of the immersion is parallel by equation (5.13). All totally real minimal isometric immersions into $\boldsymbol{H P}^{n}(1)$ with parallel second fundamental form were classified by K. Tsukada [13]. There are two possible types of such immersions, which are denoted as (R-R)-type and (R-C)-type (Proposition 3.2, [13]). It follows from (5.14) that our immersion is not of type (R-C). Among all totally real minimal isometric immersions of type (R-R) with parallel second fundamental form only $\phi_{1}, \phi_{2}, \phi_{3}, \phi_{4}, \phi_{5}$ are $\frac{1}{\sqrt{12}}$ isotropic. This completes the proof of Theorem 3 .

\section{REFERENCES}

[1] S.-S. Chern, M. Do Carmo AND S. Kobayashi, Minimal submanifolds of a sphere with second fundamental form of constant length, Functional Analysis and Related Fields, Springer-Verlag, Berlin and New York, (1970), 59-75.

[2] S. FunABAshi, Totally real submanifolds of a quaternionic Käehlerian manifold. Kodai Math. Sem. Rep. 29 (1978), 261-270.

[3] S. Funabashi, Totally complex submanifolds of a quaternionic Kählerian manifold, Kodai Math. J. 2 (1979), 314-336.

[4] H. Gauchman, Minimal submanifolds of a sphere with bounded second fundamental formn, Tras. Amer. Math. Soc. 298 (1986), 779-791.

[5] H. Gauchman, Pinching theorems for totally real minimal submanifolds of $C P^{n}(c)$, to appear in Tohoku Math. J.

[6] S. IshihARA, Quaternion Kählerian manifolds, J. Diff. Geom. 9 (1974), 483-500.

[7] N. MoK AND J.Q. ZHANG, Curvature characterization of compact Hermitian symmetric spaces, J. Diff. Geom. 23 (1986), 15-67.

[8] H. NAKAgawa AND R. TAKAgi, On locally symmetric Käehler submanifolds in a complex projective space, J. Math. Soc. Japan 28 (1976), 638-667.

[9] B. O' NeIll, Isotropic and Kähler immersions, Canad. J. Math. 17 (1965), 907-915.

[10] A. Ros, Positively curved Kähler submanifolds, Proc. Amer. Math. Soc. 93 (1985), 329-331.

[11] A. Ros, A characterization of seven compact Kähler submanifolds by holomorphic pinching, Annals of Math. 121 (1985), 377-382.

[12] J. Simons, Minimal varieties in Riemannian manifolds, Annals of Math. (2) 88 (1968), 62-105.

[13] K. Tsukada, Parallel submanifolds in a quaternion projective space, Osaka J. Math. 22 (1985), 187-241.

Department of Mathematics
Eastern Illinois University
Charleston, IL 61920
Department of Mathematics

UNIVERSITY OF ILLINOIS

URBANA, IL 61801 